\title{
Intraductal Papillary Neoplasm of the Bile Duct: A Case Report
}

ISSN: 2637-7632

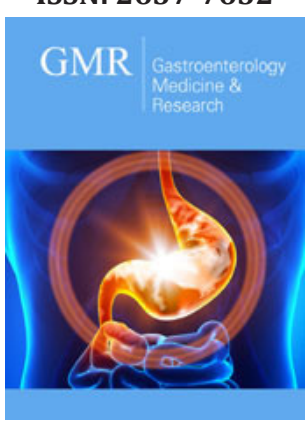

*Corresponding author: Jun Lu, Department of Hepatobiliary Surgery, East Branch of Shandong Provincial Hospital, Chinal

Submission: 悳 October 21, 2019

Published: 留 November 01, 2019

Volume 3 - Issue 5

How to cite this article: Ang Li, Niu Z, Yang $\mathrm{F}$, Xu Zhou and Jun Lu. Intraductal Papillary Neoplasm of the Bile Duct: A Case Report. Gastro Med Res. 3(5). GMR.000574. 2019.

DOI: 10.31031/GMR.2019.03.000574

Copyright@ Jun Lu, This article is distributed under the terms of the Creative Commons Attribution 4.0 International License, which permits unrestricted use and redistribution provided that the original author and source are credited.

\author{
Ang Li, Niu Z, Yang F, Xu Zhou and Jun Lu* \\ Department of Hepatobiliary Surgery, China
}

\section{Introduction}

Intraductal papillary neoplasm of the bile duct (IPN-B) is a rare tumor of the biliary system that can secrete mucus and is considered to be a precancerous lesion with high malignant potential, characterized by intraductal growth and easy recurrence. In this study, a case of IPN-B was reported.

\section{Case Report}

On January 16, 2018, a 76-year-old male patient was admitted due to "upper abdominal pain and discomfort for over 1 week". The patient presented with mild jaundice, no rigor and hyperpyrexia. Laboratory tests: glutamyl transpeptidase (GGT): 578U/L; alkaline phosphatase (ALP): 149U/L; Total bilirubin: 25.20umol/L; Indirect bilirubin: 20.10umol/L, CA125:8.39U/ ml, CA199:12.99U/ml. Imaging examination: abdominal enhancement MR \& MRCP: nodular abnormal signal lesion about $1.7 \times 1.1 \mathrm{~cm}$ was observed in the left hepatic duct near the hepatis porta, and enhancement was observed in the enhanced scan; the upstream intrahepatic bile duct in the left lobe of the liver was significantly expanded. Intraoperative findings: The patient underwent left hemi hepatectomy \& cholecystectomy. During the operation, there was free tumor embolus at the broken end of left hepatic duct. It was decided to perform common bile duct exploration. The upper segment of common bile duct was incised and removed the tumor. T-tube drainage was left in place after performing the choledochoscopy. Postoperative pathology: (left half liver and contents of bile duct cavity) intraductal papillary neoplasm, high grade, with total lesion area of about $1.1 \times 0.7 \mathrm{~cm}$. Liver resection margin, gallbladder neck lymph node (1) and "Group 12" lymph node (3) showed no tumor. The patient was followed up for over 1 year after the surgery, without obvious abnormality (Figure 1).

\section{Discussion}

IPN-B often occurs in left intrahepatic and common bile duct in patients aged 50-70 years in the Far East. It is associated with local endemicity of intrahepatic bile duct stones and clonorchiasis. The current reports are sporadically distributed and show different gender predominance in different regions [1]. The most common clinical manifestation of IPN-B patients is right upper quadrant pain, followed by recurrent acute cholangitis, chills and high fever together with obstructive jaundice symptoms, However some patients may be asymptomatic [2]. Morphologically, it is characterized by solitary or multiple gray-brown, friable, papillary masses distributed along the biliary tract. Microscopic shows epithelial proliferation in the dilated bile duct, which is papillary or tubular. In the papilla, a fine fibrovascular core can be seen. Epithelial cells are columnar or cuboidal in shape in a single layer. The nucleus is round or oval and located at the base. Few findings have been reported in laboratory tests for IPN-B. The most common finding on imaging studies is biliary dilatation, followed by intraductal masses [3]. If considered, the diagnosis can be further confirmed by means of magnetic resonance cholangiography (MRCP) or endoscopic retrograde cholangiography (ERCP). However, it is sometimes difficult to make an accurate diagnosis even if the MRCP examination is perfected. The preoperative diagnosis in the present case, for example, was considered to be left intrahepatic cholangiocarcinoma (Figure 2). In addition, There are also necessary to identify, for instance, recurrent suppurative cholangitis with bile duct stones [1], and hepatic mucinous cystic neoplasm (HMCN) [4]. 

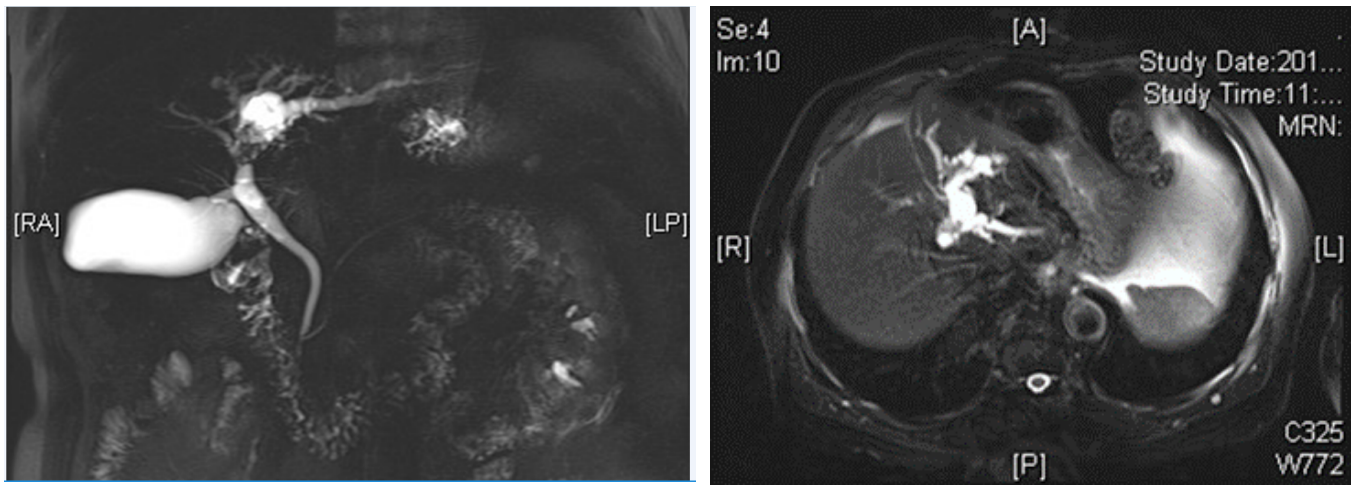

Figure 1: MR + MRCP showing nodular filling defect in the left hepatic duct and the confluence of left and right hepatic ducts in the hilar region, and biliary dilatation in the left lobe of the liver.

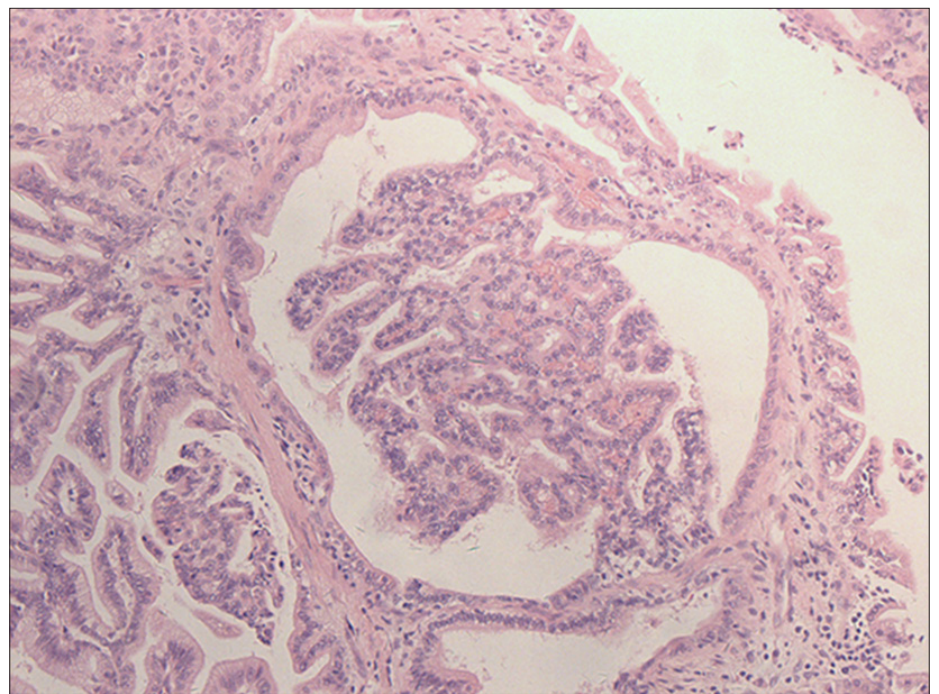

Figure 2: $200 \times$, The epithelial cells in the bile duct proliferated and showed papillary morphology. A fibrovascular core was seen in the papilla.

IPN-B has a risk of transformation to invasive cancer, and the recurrence rate is relatively high. Recent studies recommend radical resection for IPN-B patients [5] and it is the main factors affecting the prognosis of patients. Research [6] showed that: the 1-year tumor recurrence rate of such patients is $12.5 \%$, and the overall survival rate is $96.2 \%$; the 5 -year tumor recurrence rate is $47.0 \%$, and the overall survival rate is $68.8 \%$. In this case, the patient underwent R0 resection (left hemi hepatectomy \& lymph node dissection). For patients who may not be able to undergo R0 resection, liver transplantation with total biliary resection is feasible to achieve curative effect [7]. Additionally, the primary location of the tumor contributed to the patient prognosis [8]. Patients with intrahepatic IPN-B had a better postoperative survival time than those with extrahepatic IPN-B. Other factors affecting prognosis are the degree of lymph node metastasis and whether mucus is secreted. To detect recurrence as early as possible, MRCP is recommended to schedule follow-up every 3 months during the first year and every 6 months during the second year after R0 resection [9].

\section{Conclusion}

In conclusion, IPN-B is a rare tumor of the biliary tract with special pathogenesis and unclearly progression mechanisms.
Radical resection is the most effective treatment and favorable factor for long-term survival, especially in patients with early-stage IPN-B.

\section{References}

1. Wan XS, Xu YY, Qian, Yang XB, Wang AQ et al. (2013) Intraductal papillary neoplasm of the bile duct. World J Gastroenterol 19(46): 8595-8604.

2. Zheng Q, Ruan SM, Shan QY, Xu M, Chen LD, etal.(2019) Clinicopathological findings and imaging features of intraductal papillary neoplasm of the bile duct: Comparison between contrast-enhanced ultrasound and contrast-enhanced computed tomography. Abdom Radiol (NY) 44(7): 2409-2417.

3. Lou JY, Su W, Wei SM, Huang FB, Chen W, et al. (2018) [Clinical features, diagnosis and treatment of intraductal papillary neoplasm of the bile duct]. Zhonghua wai ke za zhi [Chinese Journal of Surgery] 56(5): 350354.

4. Tan Y, Milikowski C, Toribio Y, Singer A, Rojas CP, et al. (2015) Intraductal papillary neoplasm of the bile ducts: A case report and literature review. World J Gastroenterol 21(43): 12498-12504.

5. Luvira V, Pugkhem A, Bhudhisawasdi V, Pairojkul C, Sathitkarnmanee E, et al. (2017) Long-term outcome of surgical resection for intraductal papillary neoplasm of the bile duct. J Gastroenterol Hepatol 32(2): 527 533. 
6. Kim WJ, Hwang, Lee YJ, Kim KH, Park KM, et al. (2016) Clinicopathological features and long-term outcomes of intraductal papillary neoplasms of the intrahepatic bile duct. J Gastrointest Surg 20(7): 1368-1375.

7. Le DK, Agarwal A (2019) Intraductal papillary neoplasm of the bile duct Proc (Baylor University Medical Center) 32(1): 124-125.

8. You Y, Choi SH, Choi DW, Heo JS, Han IW, et al. (2019) Recurrence after resection for intraductal papillary neoplasm of bile duct (IPNB) according to tumor location. J Gastrointest Surg doi: 10.1007/s11605019-04235-8.

9. Li T, Ji Y, Zhi XT, Wang L, Yang XR, et al. (2009) A comparison of hepatic mucinous cystic neoplasms with biliary intraductal papillary neoplasms. Clin Gastroenterol Hepatol 7(5): 586-593. 